\title{
Ciclo reproductivo del molusco Atrina maura (Pterioidea: Pinnidae) en un sistema lagunar costero, al sur del Pacífico tropical mexicano
}

\author{
Claudia Angel-Pérez ${ }^{1}$, Saúl J. Serrano-Guzmán ${ }^{2} \&$ Miguel Angel Ahumada-Sempoal $^{3}$ \\ Universidad del Mar. A. P. 47. Puerto Angel, Oaxaca, 70902 México. Tel. Fax: (958) 5843049 y 78. \\ 1. shenni10@hotmail.com; 2.sserrano@angel.umar.mx; 3. ahumada@angel.umar.mx
}

Recibido 17-VI-2002. Corregido 11-VIII-2006. Aceptado 12-IV-2007.

\begin{abstract}
Reproductive cycle of the mollusk Atrina maura (Pterioidea: Pinnidae) in a coastal lagoon system of the Mexican South Pacific. From February 1997 to February 1998, random samples of the mollusk Atrina maura were collected on a monthly basis from the Corralero-Alotengo lagoon system, Oaxaca, Mexico. The soft parts were separated from the valve, washed in situ, and placed in a Davison solution. The gonadosomatic index (GSI) and the muscle yield index (MYI) were measured, and the reproductive cycle was characterized by histological cuts. A. maura has two important reproductive periods, one from April to July and another from October to November; there is a resting period from August to September. The reproductive cycle has a direct relationship with the GSI and a reverse relationship with the MYI. There was evidence of a close relationship of the spawning and post-spawning periods with the water temperature $(R=0.991, p \geq 0.002)$. Females dominate numerically throughout the year, but the difference is significant $\left(\chi^{2}: \mathrm{p} \geq 0.05\right)$ only in November. Rev. Biol. Trop. 55 (3-4): 839-852. Epub 2007 December, 28.
\end{abstract}

Key words: Atrina maura, reproductive cycle, gonadosomatic index, muscle yield index, coastal lagoon.

El molusco bivalvo Atrina maura (Sowerby, 1835) y otros miembros de la familia Pinnidae son comúnmente conocidos en México como "callo de hacha". Estos Pinnidae han tenido gran importancia pesquera en los estados del noroeste de México, y aunque en los estados del sur su explotación es menor, no deja de ser importante en Michoacán, Jalisco, Guerrero y Oaxaca (Anónimo 1994). El músculo abductor de estos organismos o "callo", tiene gran aceptación en el mercado local, nacional e internacional, razón por la que se ha incrementado la presión pesquera que junto con perturbaciones naturales (ciclones y tormentas tropicales) y alteraciones antropogénicas sobre las poblaciones silvestres o sobre el hábitat lagunar (contaminación) están disminuyendo la existencia del recurso, al punto que casi desaparece de varias zonas del Pacífico Mexicano (Arizpe y Félix 1986).
De 1997 a 1999 los Pinnidae del sistema lagunar Corralero-Alotengo, Oaxaca, adquirieron mayor importancia pesquera, debido al establecimiento de poblaciones relativamente nuevas y abundantes (Ahumada-Sempoal et al. 2002), que coincidieron con la disminución de las poblaciones ostrícolas que formaba parte importante de la pesquería de moluscos en la región. De acuerdo con Ahumada (1998) en este sistema lagunar habitan dos especies, $A$. maura y Pinna rugosa, siendo la primera dominante con el $94 \%$ de la abundancia relativa.

Con relación a los aspectos reproductivos de los Pinnidae, a nivel mundial se encuentran los estudios realizados en China por Yongqiang y Xiang (1988) sobre la ultra estructura de los oocitos de Atrina pectinata, en el Mar Mediterráneo el de Gaulejac (1995) sobre el hermafroditismo y Gaulejac et al. (1995 a,b) sobre la ultraestructura de la gametogénesis 
de Pinna nobilis. En Sudamérica, al norte de la Patagonía, Argentina, Gaspar et al. (2001) estudiaron el ciclo reproductivo de Atrina seminuda.

En el Pacífico mexicano, Noguera de Gómez y Gómez-Aguirre (1972) así como Santiago (1981), encontraron individuos de $P$. rugosa provenientes de la Bahía de la Paz, B. C. S, maduros y en desove durante primavera (marzo-abril) y verano (julio-septiembre), presentando hermafroditismo aislado. Mientras en la Bahía de Bacochibampo, Son, Cendejas et al. (1985) dan evidencias de una relación directa entre el desove de $P$. rugosa y el incremento de la temperatura del mar.

En Bahía Magdalena, B. C. S., RodríguezJaramilo et al. (2001) recolectaron organismos gonocóricos de $A$. maura, con proceso gametogénico y desove sincrónico principalmente de enero a marzo. En la costa del Pacífico tropical mexicano, Aguilar (1964) detectó en A. maura de la Bahía de Acapulco, Gro., dos periodos reproductivos, uno de noviembre a enero y otro de abril a junio, al igual que Soria (1989) en la desembocadura del Río Balsas, Mich., donde además encontró individuos sexualmente maduros todo el año y observó que individuos de $16 \mathrm{~cm}$ de longitud valvar alcanzaron la madurez sexual, mientras que dentro del sistema lagunar Corralero-Alotengo, Oax., Ahumada-Sempoal et al. (2002) indicaron que individuos de A. maura, de $10 \mathrm{~cm}$ de largo, tuvieron su primera maduración sexual. Por otra parte Leyva-Valencia et al. (2001) determinaron la tolerancia a la salinidad y temperatura máxima superior, así como la temperatura óptima para el crecimiento de A. maura.

El objetivo de nuestro trabajo fue reconocer histológicamente las etapas del desarrollo gonádico de A. maura y caracterizar su actividad reproductiva durante el ciclo anual de 1997-98, estableciendo su relación con el índice gonadosomático, el índice de rendimiento muscular y la temperatura del agua dentro del canal que comunica al sistema lagunar Corralero-Alotengo con el mar adyacente.

\section{MATERIALES Y MÉTODOS}

El sistema lagunar Corralero-Alotengo se localiza en la costa oeste del estado de Oaxaca, México, entre las coordenadas: $16^{\circ} 11^{\prime} 15^{\prime \prime}$ y $16^{\circ} 16^{\prime} 30^{\prime \prime}$ latitud norte y $98^{\circ} 05^{\prime} 00^{\prime \prime}$ y $98^{\circ}$ $12^{\prime} 30^{\prime}$ " longitud oeste. De acuerdo con la clasificación climática de Köppen, modificada por García (1984), el tipo de clima predominante en la región es Aw2 (w) ig, cálido subhúmedo con lluvias en verano.

Mensualmente, durante el ciclo anual comprendido de febrero de 1997 a febrero de 1998, en el canal principal que comunica al sistema lagunar con el mar adyacente, se recolectaron del bentos lagunar mediante buceo autónomo 14 organismos de Atrina maura (de 15 a $25 \mathrm{~cm}$ de longitud valvar), cuantificando paralelamente la temperatura del agua con una sonda múltiple HYDROLAB ${ }^{\circledR}$, con precisión de $\pm 0.15^{\circ} \mathrm{C}$.

Después de sacrificar a cada animal, las partes blandas (masa visceral y músculos) se desprendieron cuidadosamente de las valvas, enjuagándolas in situ con agua de mar, para ser fijadas en solución Davison durante $24 \mathrm{hr}$ y posteriormente conservarlas en alcohol etílico al 70\% (Humason 1979). En el laboratorio se pesó con una balanza analítica $( \pm 0.1 \mathrm{mg})$ toda la parte blanda, y por separado la masa visceral que incluyó la gónada (sin manto, branquias, bisu y pie) y los músculos, con el objeto de calcular en base húmeda, el índice gonadosomático (IGS) y el índice de rendimiento muscular (IRM), a través de las relaciones descritas por Marshall (1960):

$$
\begin{aligned}
& \text { IGS }=\frac{\text { Peso de la masa visceral con gónada }}{\text { Peso de las partes blandas }} \times 100 \\
& \text { e } \quad \text { IRM }=\frac{\text { Peso muscular }}{\text { Peso de las partes blandas }} \times 100
\end{aligned}
$$

Se obtuvo una sección transversal de 10 $\mathrm{mm}^{3}$ de gónada a la altura de los nefridios, y se siguieron las etapas convencionales y sucesivas de: deshidratación, aclarado, inclusión, 
corte, tinción y montaje (Humason 1979). Las etapas del desarrollo gonádico se tipificaron considerando el tamaño, cantidad, morfología y afinidad de los colorantes por cada uno de los tipos celulares; las fases que aquí se describen corresponden a las mismas que Aguilar (1964) y Soria (1989) reconocieron como: indiferenciado, gametogénesis inicial, gametogénesis avanzada, madurez, desove y postdesove.

El sexo de cada individuo se determinó observando microscópicamente sus gónadas. La proporción $(p)$ entre machos $(m)$ y hembras (h) se contrastó con una prueba de hipótesis (Ho: $p m-p h=0 ; \mathrm{Ha}: p m-p h \neq 0$; siguiendo el procedimiento descrito en Daniel 2002). La relación del ciclo reproductivo con la temperatura, se estableció mediante análisis canónico (Johnson y Wichern 1982).

\section{RESULTADOS}

Reconocimiento histológico y caracterización de las etapas del desarrollo gonádico:

\section{Indiferenciado}

Los acinos están sostenidos por escaso tejido conjuntivo (Tc) donde se observan algunos fagocitos (Fc) (Fig. 1A). Estado caracterizado por la ausencia de gametos.

\section{Hembras}

Gametogénesis inicial: Los acinos son pequeños, unos empiezan a desarrollarse mientras que otros están en recuperación después de haber participado en un desove, existe un espacio de tejido conjuntivo entre un acino y otro. Esta etapa se caracteriza por ovogonias $(\mathrm{Og})$ casi esféricas que miden en promedio 3-5 $\mu \mathrm{m}$, unidas a la pared del acino como si fueran cuentas de un collar, también están presentes algunos ovocitos vitelogénicos pedunculados (Oc) (Fig. 1B).

Gametogénesis avanzada: Los acinos han incrementado su tamaño y presentan ovocitos en diferentes etapas de desarrollo. Los ovocitos vitelogénicos que son característicos de ésta fase, tienen forma de pera y miden en promedio 45-50 $\mu \mathrm{m}$, con un pedúnculo que los mantiene unidos a la pared del acino, algunos acinos muy cercanos forman trabéculas, el tejido conjuntivo es escaso (Fig. 1C).

Madurez: Etapa caracterizada por ovocitos postvitelogénicos que han completado su desarrollo, sin pedúnculo, son casi redondos y están libres en el lumen del acino, miden hasta $60 \mu \mathrm{m}$, no existe espacio entre los acinos, ya que están completamente desarrollados (Fig. 1D). El núcleo y nucleolo se distinguen claramente y el cúmulo mitocondrial se observa teñido intensamente fuera del núcleo.

Desove: Fase caracterizada por la liberación de ovocitos que han completado su desarrollo. En un desove parcial, el lumen (L) del acino se encuentra casi vacío y los ovocitos que aún están en desarrollo se encuentran unidos a su pared. Mientras que en el desove total los ovocitos son expulsados casi totalmente, y en el lumen sólo se observan ovocitos residuales libres y algunos fagocitos (Fig. 1E).

Postdesove: Los ovocitos han sido liberados en su totalidad, los acinos se observan casi vacíos y/o en reabsorción con restos de tejido conjuntivo. Existen gametos residuales y se incrementan los fagocitos alrededor de los ovocitos liberados pero no expulsados (Fig. 1F).

\section{Machos}

Gametogénesis inicial: Estadío caracterizado por la presencia de espermatogonias (Eg) que miden en promedio $3 \mu \mathrm{m}$ y se observan en la periferia interior del acino, seguidas hacia su interior por los espermatocitos (Ec). Los acinos en desarrollo se observan en diferentes tamaños, distantes unos de otros y conectados por tejido conjuntivo (Fig. 2A).

Gametogénesis avanzada: Los acinos, más desarrollados que en la gametogénesis 

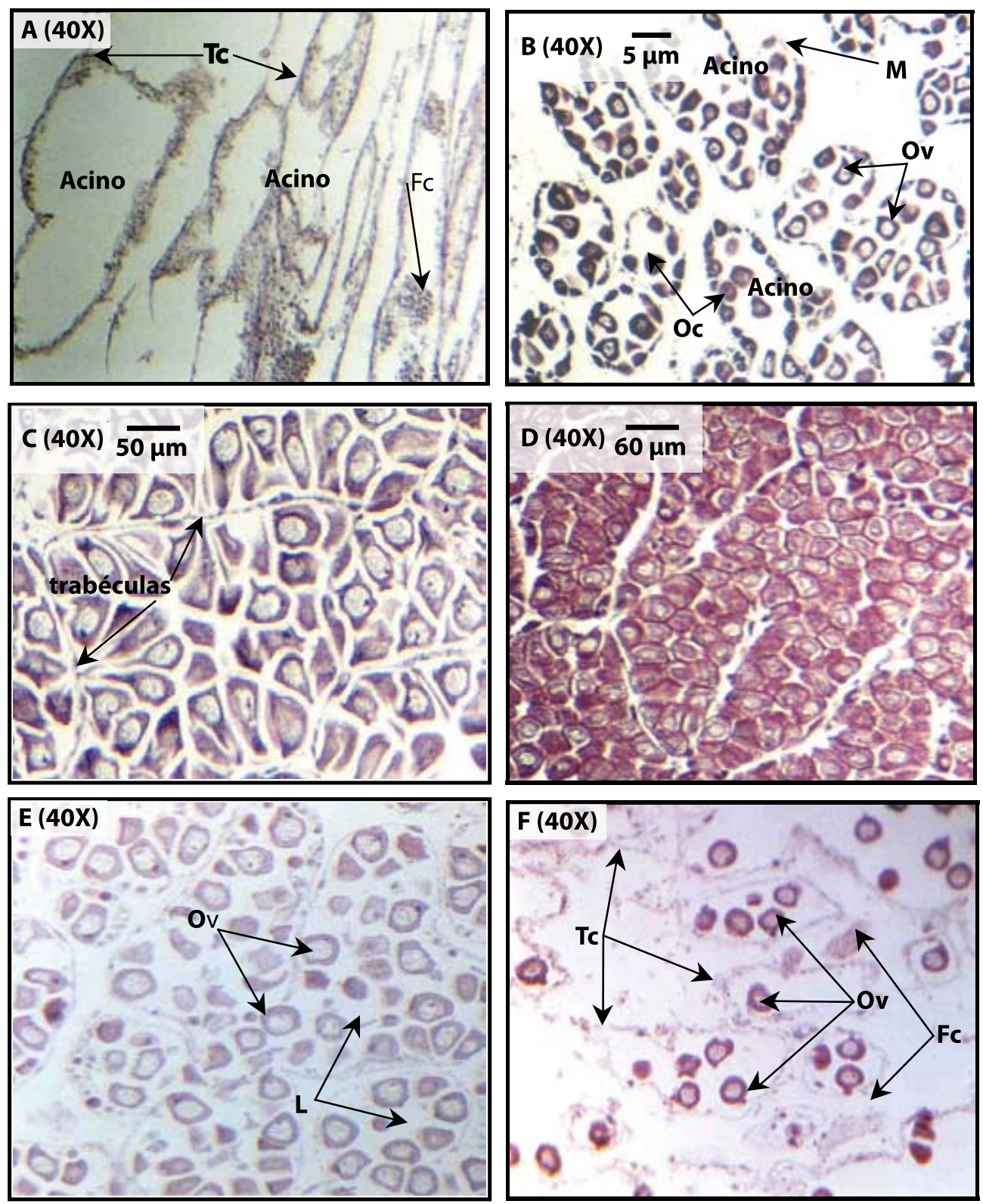

Fig. 1. Cortes histológicos en gónadas de A. maura (10x). A. Indiferenciado. B-F: Hembras; B: Gametogénesis inicial, C: Gametogénesis avanzada, D: Madurez, E: Desove, F: Post-desove. Tc= Tejido conjuntivo, Fc= Fagocitos, M= Membrana, $\mathrm{Ov}=$ Óvulos, $\mathrm{Oc}=$ Ovocitos, $\mathrm{L}=$ Lumen.

Fig. 1. Histological cuts of gonads of A. maura (10x). A. Undifferentiated. B-F: Females; B: Early active stage, C: Late active stage, D: Mature, E: Spawning, F: Post-spawning stage. $\mathrm{Fc}=$ Phagocytes, $\mathrm{L}=$ Lumen, $\mathrm{M}=\mathrm{Membrane}$, Oc= Oocytes, $\mathrm{Ov}=$ Ovules, $\mathrm{Tc}=$ Conjunctive Tissue. 

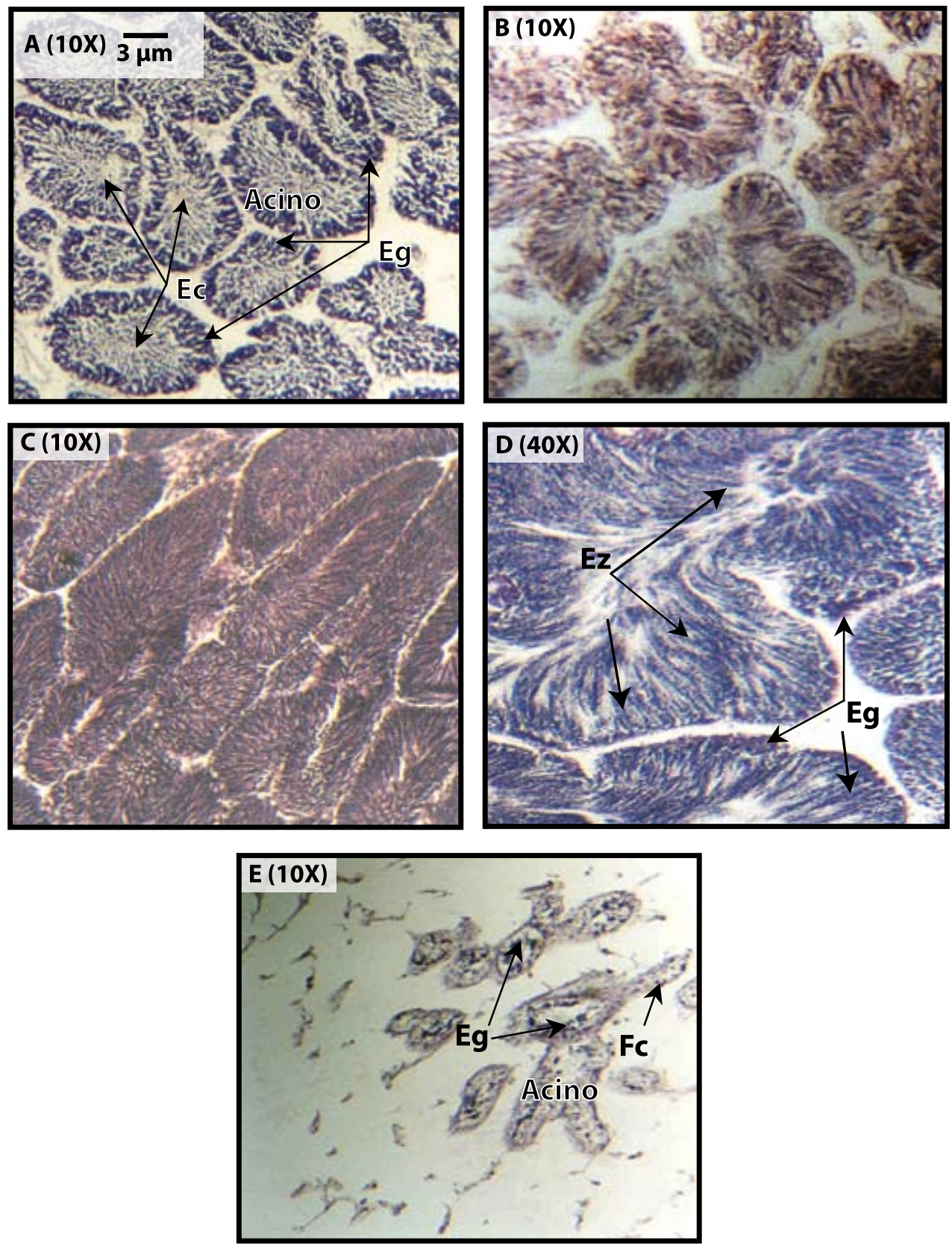

Fig. 2. Cortes histológicos en gónadas de A. maura (10x). Machos; A: Gametogénesis inicial, B: Gametogénesis avanzada, C: Madurez, D: Desove, E: Post-desove. Ec $=$ Espermatocitos, Eg= Espermatogonias, Ez $=$ Espermatozoides, $\mathrm{Fc}=$ Fagocitos.

Fig. 2. Histological cuts of gonads of A. maura (10x). Males; A: Early active stage, B: Late active stage, C: Mature, D: Spawning, E: Post-spawning stage. Ec= Spermatocytes, Eg= Spermatogonia, Ez= Spermatozoa, Fc= Phagocytes. 
inicial tienen varias capas de células. Las espermatogonias van disminuyendo y se incrementa el número de espermatocitos, algunos espermatozoides dejan ver sus flagelos, hacia el lumen del acino (Fig. 2B).

Madurez: Los acinos están completamente desarrollados, no existe espacio entre ellos, ni se observa tejido conjuntivo, disminuyen las espermatogonias y los espermatocitos, y se observan abundantes espermatozoides (Fig. 2C).

Desove: En el desove parcial el lumen del acino se encuentra con partes vacías, debido a la expulsión de espermatozoides (Ez), mientras que el resto presenta espermatocitos y algunas espermatogonias hacia la pared del acino (Fig. 2D). En el desove total los acinos están vacíos o bien con algunos gametos residuales.

Postdesove: Los acinos se observan con algunas espermatogonias y gametos residuales, que son reabsorbidos por las células fagocíticas (Fig. 2E).

\section{Observaciones particulares dentro del ciclo reproductivo}

Células auxiliares: Durante la vitelogénesis estas células se observan unidas a los ovocitos y en la postvitelogénesis se encuentran libres en el lumen del acino (Fig. 3A).

Atresias: Pueden presentarse durante la vitelogénesis, así como en la postvitelogénesis donde han perdido su forma redondeada, miden $40 \mu \mathrm{m}$ aproximadamente. Se observaron principalmente en el mes de noviembre (Fig. 3B), posterior al paso de los huracanes "Pauline" (8/X/1997) y "Rick" (9/XI/1997).

Organismo al momento de desovar (ejemplar del 1 de junio de 1997): La hembra analizada tuvo todos los ovocitos libres en el lumen del acino y totalmente separados unos de otros (Fig. 3C). Según describe Lora-Vilchis et al. (1997) los ovocitos están bien formados, sin vesícula germinal (núcleo).
Hermafrodita: Se observó únicamente un ejemplar en etapa de postdesove (el 15 de septiembre de 1997) con gametos tanto de macho como de hembra (Fig. 3D).

Ciclo reproductivo: Atrina maura mostró actividad gametogénica durante todos los meses del año, con periodos de desove de abril a julio y de octubre a noviembre de 1997, así como en enero de 1998, siendo los meses más importantes abril y octubre (Fig. 4). Los individuos de la muestra analizada maduraron en febrero (1998), marzo y mayo (1997), siendo marzo el mes más importante (representado por el $90 \%$ de los organismos) (Fig. 4). La gametogénesis (inicial y avanzada) estuvo presente casi todo el año, llegando a ser predominante durante el mes de diciembre (en el $90 \%$ de los individuos analizados) (Fig. 4). La condición de postdesove se observó de abril a septiembre, con dos meses importantes en junio y agosto (representando al $40 \%$ de la población), mientras que individuos indiferenciados se observan de julio a septiembre, pero sobre todo en agosto (40\% de los ejemplares) (Fig. 4).

Debido a la persistente actividad reproductiva de A. maura, pueden encontrarse organismos en diferentes etapas de desarrollo gonadal, algunos desovan y al mismo tiempo producen nuevos gametos, es decir, sólo pasan por las fases de recuperación (gametogénesis inicial y avanzada), madurez y desove (Fig. 5); mientras que otros miembros de la población reproductiva realizan el ciclo completo: gametogénesis, madurez, desove, postdesove e indiferenciación (Fig. 5).

Índice gonadosomático e índice de rendimiento muscular: El IGS tuvo los valores más bajos en los meses de agosto (15.7\%) y septiembre (16.2\%), alcanzando los valores más altos en junio (33\%) y noviembre (39\%), cuando el peso de la gónada y del sistema digestivo oscilaron entre 2.4 y 35.3 g respectivamente. De acuerdo con este índice, A. maura está en gametogénesis y madurez (ascenso de valores) de abril a junio, de octubre a noviembre y en enero; mientras que se deduce 

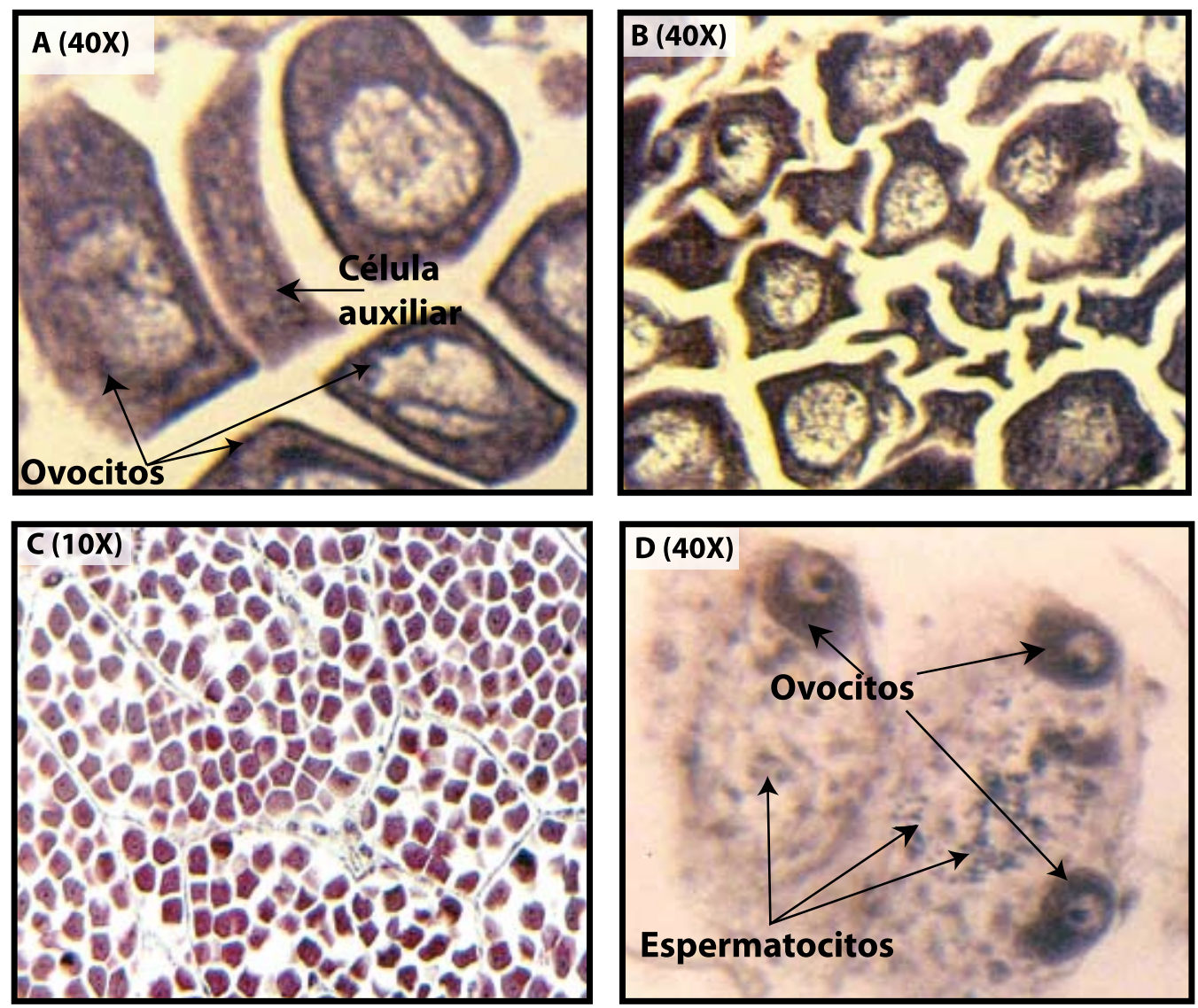

Fig. 3. Observaciones particulares del ciclo reproductivo de A. maura (10x). A: Célula Auxiliar, B: Atresias, C: Desove, D: Hermafrodita.

Fig. 3. Some features of the reproductive cycle of A. maura (10x). A: Auxiliary cell, B: Atresias, C: Spawning, D: Hermaphrodite.

actividad de desoves (descenso de los valores) de julio a septiembre, en diciembre y de febrero a marzo (Fig. 4). Por su parte el IRM, indica que los músculos tienden a aumentar su peso de abril a mayo, de agosto a septiembre y de enero a febrero, y disminuyen en marzo, de junio a julio y de octubre a diciembre (Fig. 4). Los valores más altos se registraron en agosto $(34.5 \%)$ y septiembre $(38.9 \%)$ y los más bajos en marzo y diciembre $(23.5-23.0 \%)$. El intervalo de peso muscular vario de 23.2 a $38.6 \mathrm{~g}$ en individuos con tallas de 15 a $25 \mathrm{~cm}$ de longitud valvar.
Relación de la temperatura del agua con el ciclo reproductivo: La temperatura del agua inició su ascenso a partir de febrero de 1997 $\left(28.2^{\circ} \mathrm{C}\right)$, para alcanzar su máximo en agosto de $1998\left(30.8{ }^{\circ} \mathrm{C}\right)$, cuando el análisis histológico y el IGS indicaron que los organismos se encontraban en fases de post-desove e indiferenciados, coincidiendo con altos valores del IRM (Fig. 4). Entre tanto, el descenso paulatino de la temperatura, de septiembre a diciembre de 1997, permitió observar animales en procesos de gametogénesis; mientras que las etapas de 


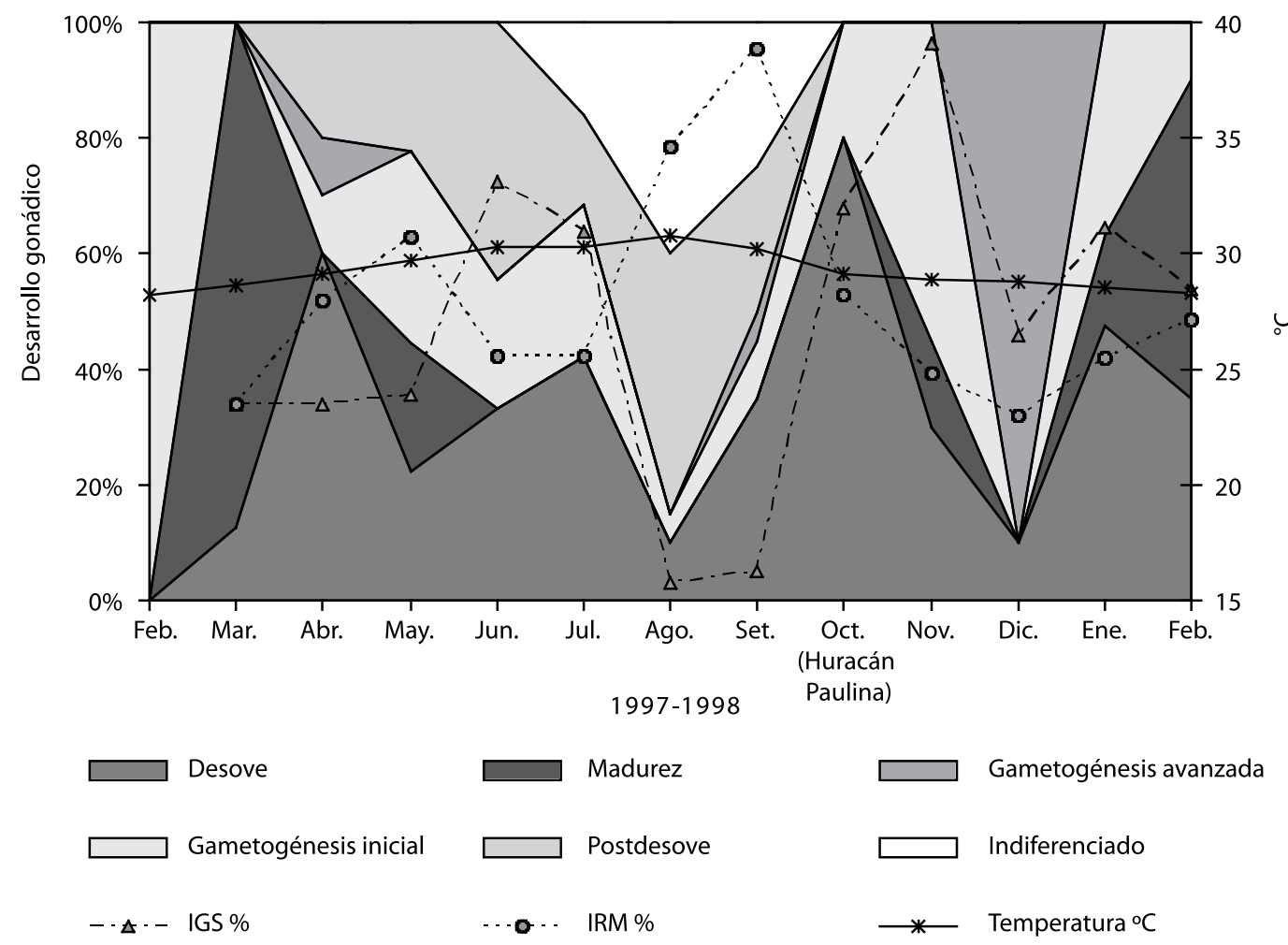

Fig. 4. Ciclo reproductivo de A. maura en el sistema lagunar Corralero-Alotengo, Oaxaca, México.

Fig. 4. Reproductive cycle of A. maura in the Corralero-Alotengo lagoon system, Oaxaca, Mexico.

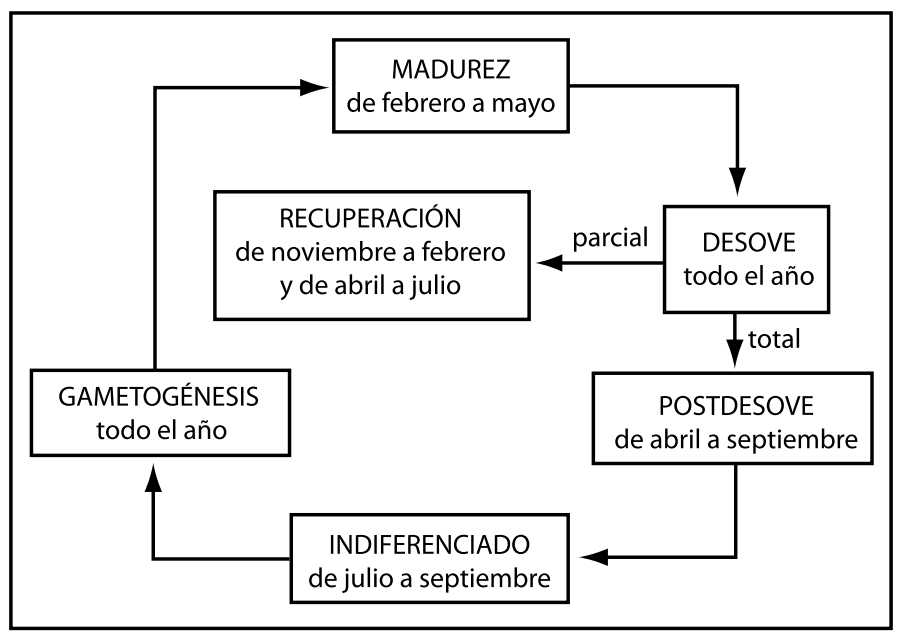

Fig. 5. Estrategias reproductivas de A. maura en el sistema lagunar Corralero-Alotengo, Oaxaca, México.

Fig. 5. Reproductive strategies of A. maura in the Corralero-Alotengo lagoon system, Oaxaca, Mexico. 
madurez y desove, coincidieron con temperaturas de 28.5 a $29.2{ }^{\circ} \mathrm{C}$ (excepto en junio cuando fue de 30.2). La temperatura continuó descendiendo hasta llegar a $28.3{ }^{\circ} \mathrm{C}$ en febrero de 1998 , concordando con gran actividad gametogénica y bajos valores del IRM (Fig. 4). De acuerdo con el análisis canónico esta variable ambiental tiene una estrecha relación con el ciclo reproductivo $(\mathrm{R}=0.991, \mathrm{p} \geq$ 0.002 ), sobre todo con las fases de post-desove $(86 \%)$, desove $(84 \%)$ e indiferenciación sexual $(77 \%)$.

\section{Proporción de sexos}

En la muestra analizada (14 organismos mensuales) del sistema lagunar CorraleroAlotengo se obtuvo durante casi todo el año una proporción que favoreció la dominancia relativa de hembras sobre machos, excepto en febrero y mayo cuando los machos dominaron hasta en un $30 \%$. Sin embargo, la proporción de machos y hembras sólo tuvo diferencia estadística significativa en el mes de noviembre $\left(\chi^{2}: p \geq 0.05\right)$. En cuanto a los organismos indiferenciados, su presencia se limitó a los meses de julio (15\%), agosto (40\%) y septiembre (29\%) (Fig. 6).

\section{DISCUSIÓN}

La población bentónica de A. maura dentro del sistema lagunar Corralero-Alotengo, mantuvo continua actividad gametogénica y desovó durante casi todo 1997, hechos que se

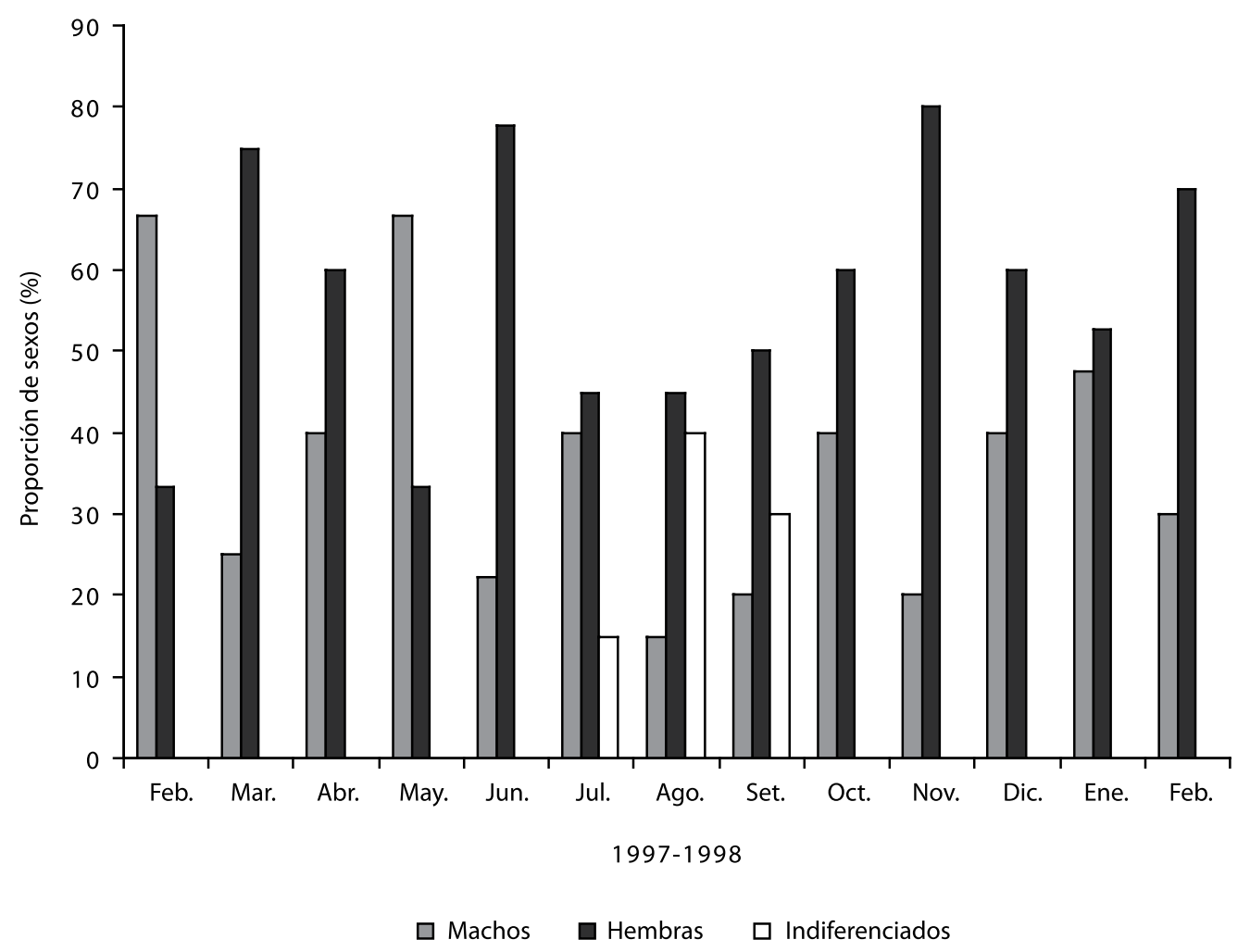

Fig. 6. Proporción sexual de A. maura en el sistema lagunar Corralero-Alotengo, Oaxaca, México.

Fig. 6. Sexual ratio of A. maura in the Corralero-Alotengo lagoon system, Oaxaca, Mexico. 
corroboraron mediante el análisis histológico de sus gónadas, identificando como importantes periodos de desoves masivos, los meses de abril a julio, cuando del 60 al $42 \%$ de la población se encontraba expulsando gametos al ambiente lagunar, así como de septiembre a noviembre (35 a $80 \%$ ) y en menor proporción durante enero y febrero de 1998 (47 a 35\%) (Fig. 4). Se reconoció un periodo de reposo reproductivo de julio a septiembre, cuando del 16 al $45 \%$ de la población se encontraba en estado de postdesove y 16 a $40 \%$ estaba indiferenciada, lo que significa que aproximadamente del 32 al $85 \%$ de la población no se encontraba realizando actividad reproductiva. El ciclo reproductivo se interrumpió en diciembre (Fig. 4), como respuesta al paso de los huracanes "Pauline" (8 de octubre de 1997) y "Rick" (9 de noviembre de 1997), quienes provocaron lluvias torrenciales y gran acarreo de terrígenos, modificando la salinidad del sistema lagunar (Ahumada y Ruiz 2000), e induciendo la formación de atresias en las gónadas de $A$. maura (Fig. 3B y 4). Este proceso degenerativo de los ovocitos, es comúnmente observado en especies cuya gametogénesis está bajo el control de las condiciones ambientales (Gaulejac 1995a,b).

El ciclo reproductivo de $A$. maura, fue similar al reconocido por Aguilar (1964) en Acapulco, Gro. y por Soria (1989) en la desembocadura del Río Balsas, Mich. El primer periodo reproductivo coincidió con el provisto por ambos autores (de abril a julio), mientras que el segundo se adelantó un mes (de noviembre a octubre) y de acuerdo con Ahumada-Sempoal et al. (2002) involucró principalmente a individuos de 13.5 a $21.5 \mathrm{~cm}$ de longitud valvar, con una marcada estacionalidad en individuos mayores a $22 \mathrm{~cm}$, quienes reactivan su gametogénesis inicial en abril y diciembre (Fig. 4). De manera que se identificaron dos estrategias reproductivas: una con desoves parciales presumiblemente de organismos jóvenes, que inician su reclutamiento a la población reproductiva (en julio y enero) y otra con desoves totales más sincrónicos, donde participan masivamente los individuos que ya componen la población reproductiva (en abril y octubre) (Fig. 4). Por lo tanto, de acuerdo con Gorrostieta (1997) y Prado (1998), puede hablarse de un semiciclo de reproducción con desoves parciales, dentro de otro ciclo completo (Fig. 5).

Ciclos reproductivos como el de A. maura, han sido reconocidos en otras especies que habitan regiones geográficas con pequeños cambios estacionales, como las zonas tropicales y sitios con mínimas fluctuaciones ambientales como las profundidades marinas, donde la reproducción es continua (Giese y Pearse 1974, Sastry 1979, Mackie 1984). Sin embargo, Rodríguez-Jaramilo et al. (2001) observaron en Bahía Magdalena, B. C. S. solo un periodo reproductivo con desove sincrónico de enero a marzo, dando evidencia de que ésta especie que habita diferentes regiones biogeográficas, tiene gran plasticidad reproductiva, adaptándose al ambiente local, ya que la reproducción es una respuesta endógena genéticamente controlada por el ambiente, conocida como gradiente latitudinal de reproducción (Sastry 1970, 1979).

En A. maura los dos picos de mayor actividad gametogénica o desoves importantes se activaron cuando la temperatura alcanzó $29.2^{\circ} \mathrm{C}$ tanto en abril como en octubre, coincidiendo con el incremento y decremento de la temperatura del agua (Fig. 4), mientras que el período vegetativo o de reposo ocurrió durante la temporada más calida del año (de julio a septiembre, a 30.3 - $30.8^{\circ} \mathrm{C}$ ). Estos hechos, no coincide del todo con lo observado en otras especies de bivalvos, ya que de acuerdo con Loosanoff y Davis (1963) y Sastry (1963), la respuesta reproductiva depende de la especie y del área geográfica, por lo que, mientras algunas especies desovan cuando la temperatura va en aumento, otras lo hacen cuando la temperatura alcanza su máximo o cuando va en descenso. Según Rodríguez-Jaramilo et al. (2001), la temperatura es un factor importante en la activación del ciclo reproductivo de $A$. maura y determinante para alcanzar la madurez e inducir el desove, como lo comprobaron SerranoGuzmán y Robles-Mungaray (1996) al realizar en un criadero comercial, el acondicionamiento de progenitores para inducir térmicamente su desove y realizar el cultivo larvario de $A$. maura. 
E igualmente demostró ser un factor regulador de las tasas metabólicas de juveniles de $A$. maura (Serrano-Guzmán 1997).

A pesar de lo anterior, se sabe que la temperatura no es el único factor importante en la regulación del ciclo reproductivo, pues existen otros: como el estado fisiológico de los individuos, la abundancia, calidad y disponibilidad de alimento (Sastry 1968), la duración del fotoperiodo y la periodicidad lunar (Giese y Pearse 1974, Mackie 1984). Sin embargo, la temperatura ha sido catalogada como el factor detonante más importante (Orton 1920, Loosanoff y Davis 1952, Gunter 1957, Vernber 1962, Kinne 1963, 1964, McWhinne 1967, Helgpeth y Gonor 1969) por lo que, la influencia que puede tener sobre el ciclo reproductivo de $A$. maura no es única, pero es determinante para el desove, e incluso guarda estrecha relación con las dos etapas subsecuentes (postdesove - 86\% - e indiferenciado - 77\% -), como lo evidenció el análisis canónico.

El índice gonadosomático no fue un buen estimador de la actividad gametogénica de $A$. maura, debido a que los cambios del IGS no describieron fidedignamente la tendencia del ciclo reproductivo caracterizado mediante el análisis histológico de sus gónadas, ya que cuando los organismos estaban en gametogénesis, madurez y desove, los valores del IGS se observaron ligeramente desfasados (Fig. 4). Sin embargo, durante agosto y septiembre, cuando ocurrió la inactividad gonádica (etapas de indiferenciado y post-desove) si coincidieron con los valores más bajos del IGS (15.7-16.2\%) (Fig. 4). Por lo que el uso del IGS como descriptor inequívoco del ciclo reproductivo de A. maura debe considerar sus limitaciones y tomarse con precaución. Además, cabe recordar que la gónada esta íntimamente relacionada con la glándula digestiva, lo que junto con el contenido de agua, probablemente influyó en las evaluaciones. Con respecto a las fases del desarrollo gonádico y el índice de rendimiento muscular, se observó una relación inversa, ya que los valores más altos del IRM se obtuvieron en agosto $(34.5 \%)$ y septiembre $(38.9 \%)$, cuando los organismos estaban indiferenciados y en postdesove, y los más bajos cuando las etapas reproductivas predominantes fueron la gametogénesis, madurez y desove (Fig. 4). Es posible que esta relación se deba a que durante el periodo de gametogénesis intensa, el costo energético de la producción de gametos, que frecuentemente excede a la energía asimilada (Le Pennec et al. 1991), esta subsidiado por la reabsorción del músculo abductor, el sitio de mayor reserva energética (como glicógeno) como lo han indicado Walne (1970) y Sastry (1979), lo que provocó la consecuente disminución del IRM. Por otra parte, durante la interrupción del ciclo reproductivo en diciembre, cuando el $90 \%$ de la población analizada estaba en gametogénesis inicial, tanto el IGS como el IRM decayeron rápida y sensiblemente (Fig. 4).

En Corralero-Alotengo A. maura fue reconocida como una especie gonocórica, con sexos claramente definidos. Sin embargo, se encontró un ejemplar caracterizado como hermafrodita funcional "accidental" (Fig. 3D), es decir, un individuo ocasionalmente monoico dentro de la población de una especie típicamente dioica (Mackie 1984). En otros estudios del ciclo reproductivo de los Pinnidae, se indica la presencia de hermafroditismo como casos no relevantes, ya que sólo han encontrado uno o dos ejemplares (Aguilar 1964, Noguera de Gómez y Gómez-Aguirre 1972, Santiago 1981, Soria 1989 Gaulejac 1995). En cuanto a la proporción de sexos, comúnmente predominaron las hembras sobre los machos, esta proporción en términos generales coincide con los resultados obtenidos por Soria (1989). Lo que nos conduce a pensar que, esta proporción posiblemente sirva para incrementar el éxito reproductivo, asegurando la persistencia de la especie, ya que una población con mayor número de hembras puede interpretarse como una población con mayores posibilidades de crecimiento, que aquella donde dominan los machos. Sin embargo, la proporción encontrada difiere de la referida por AhumadaSempoal et al. (2002) para A. maura en la misma población (1:1 de febrero a mayo y en septiembre, con predominancia de machos de junio a agosto). Diferencia que consideramos 
atribuible al tamaño de la muestra analizada mensualmente, que en su caso fue de cuatro a nueve veces mayor (46 a 120 organismos) a la aquí considerada (14 individuos). Razón por la que, la proporción sexual aquí descrita deberá considerarse con las limitaciones pertinentes, a fin de no extrapolarla a la población bentónica de A.maura existente en el canal principal del sistema lagunar Corralero-Alotengo.

Finalmente se sugiere que para regular la explotación racional de éste recurso, dentro del sistema lagunar Corralero-Alotengo, deberían establecerse dos periodos de veda reproductiva, uno de mayo a junio y otro de octubre a noviembre, coincidiendo con los desoves masivos más importantes.

\section{AGRADECIMIENTOS}

A Eréndira Gorrostieta Hurtado y Ma. del Carmen Rodríguez Jaramillo por su asesoría. A la Universidad del Mar (UMar) y al SIBEJ - CONACYT por el apoyo recibido a través del proyecto "Caracterización ambiental y aprovechamiento de los recursos naturales de los sistemas lagunares ChacahuaPastoría y Corralero-Alotengo", Clave: OAXRNMA-005/96. Así como al CONACYT y la SAGARPA, por el apoyo brindado a través del proyecto "Estado de salud de los moluscos bivalvos en cultivo y poblaciones silvestres de bivalvos de interés acuícola en la costa de Oaxaca", Clave: SAGARPA- CONACYT 2003 $-\mathrm{C} 01-125$.

\section{RESUMEN}

De febrero de 1997 a febrero de 1998, se recolectaron mensualmente muestras aleatorias del molusco Atrina maura en el sistema lagunar Corralero-Alotengo, Oaxaca, México. Se extrajeron las partes blandas para lavarlas in situ y fijarlas en solución Davison. En el laboratorio se pesaron para obtener los índices gonadosomático (IGS) y de rendimiento muscular (IRM), y mediante cortes histológico de las gónadas se caracterizó el ciclo reproductivo. Esta población se reproduce casi todo el año, con dos periodos reproductivos importantes: uno de abril a julio y otro de octubre a noviembre, y una sola época de reposo, en agosto y septiembre. El ciclo reproductivo tiene una relación directa con el IGS e inversa con el IRM. Hay evidencias de una relación estrecha de las época de desove y postdesove con la temperatura del agua $(\mathrm{R}=0.991, \mathrm{p} \geq$ 0.002). Las hembras predominaron casi todo el año, aunque esta proporción solo tuvo diferencias estadísticamente significativas en noviembre $\left(\chi^{2}: p \geq 0.05\right)$

Palabras clave: Atrina maura, ciclo reproductivo, índice gonadosomático, producción muscular, laguna costera.

\section{REFERENCIAS}

Aguilar, I.F. 1964. Contribución al estudio histológico de las gónadas de Atrina maura Sowerby, 1835 (Mollusca: Pinnidae). Tesis de licenciatura en Biología, Escuela Nacional de Ciencias Biológicas, Instituto Politécnico Nacional, México D.F., México.

Ahumada, S.M. A. 1998. La Familia Pinnidae (Callo de Hacha) de Oaxaca, México. Ciencia Mar 2: 42-44.

Ahumada, M.A. \& N. Ruiz. 2000. Condiciones hidrológicas del sistema lagunar Corralero-Alotengo, Oaxaca, México. Memorias del XII Congreso Nacional de Oceanografía, Huatulco, Oaxaca. Instituto Nacional de la Pesca, México D.F., México.

Ahumada-Sempoal, M.A., S.J. Serrano-Guzmán \& N. Ruiz-García. 2002. Abundancia, estructura poblacional y crecimiento de Atrina maura (Bivalvia: Pinnidae) en una laguna costera tropical del Pacífico mexicano. Rev. Biol. Trop. 50: 1091-1100.

Arizpe, O. \& R. Félix. 1986. Crecimiento de Pinna rugosa (Sowerby 1835), Bahía de la Paz, México. An. Inst. Cienc. Mar Limnol. 13: 167-172.

Anónimo, 1994. Atlas Pesquero de México. Instituto Nacional de la Pesca, México D.F., México.

Cendejas, J.M., M.G. Carvallo \& L.M. Juárez. 1985. Experimental spat collection and early growth of the pen shell, Pinna rugosa (Pelecipoda, Pinnidae) from the Gulf of California. Aquac. 48: 331-336.

Daniel, W.W. 2002. Bioestadística, bases para el análisis de las ciencias de la salud. Limusa - Wiley. México D.F., México.

García, E. 1984. Nuevo Atlas Porrúa de la República Mexicana. Porrúa, México D.F., México.

Gaspar S.R., M.S. Pascual, \& V.H. Fernández Cartes. 2001. Reproductive cycle of the cholga paleta, Atrina seminuda Lamarck, 1819 (Bivalvia: Pinnidae) from 
northern Patagonia, Argentina, J. Shellfish Res. 21: 479-488.

Gaulejac, B. 1995. Sucessive hermaphroditism of Pinna nobilis (Bivalvia: Pterioidea). Vie 318: 99-103.

Gaulejac, B., M. Henry \& N. Vicente. 1995a. An ultrastructural study of gametogenesis of the marine bivalve Pinna nobilis (Linnaeus, 1758) Vol. 1. Oogenesis. J. Moll. Stud. 61: 375-392.

Gaulejac, B., M. Henry \& N. Vicente. 1995b. An ultrastructural study of gametogenesis of the marine bivalve Pinna nobilis (Linnaeus, 1758) Vol. 2. Spermatogenesis. J. Moll. Stud. 61: 393-403.

Giese, A.C. \& J.S. Pearse. 1974. Introduction, p. 1-49. In A. C. Giese \& J. S. Pearse (eds.). Reproduction of marine invertebrates. Academic, Nueva york, Nueva York, EE.UU.

Gorrostieta, H.E. 1997. Ciclo gonádico de Arca pacifica (Sowerby, 1833) y de Cardita affinis, 1833 (Mollusca: Bibalvia) en Punta Arena, Bahía Concepción, B. C. S. Tesis de Maestría. CICIMAR. La Paz, Baja California Sur, México.

Gunter, G. 1957. Temperature. Geol. Soc. Am. Mem. 67: 159-184.

Helgpeth, J.W. \& J.J. Gonor, 1969. Aspects of the potential effects of thermal alteration on marine and estuarine benthos. In P. A. Krenkel \& F. L. Parker (eds.). Biological aspects of thermal pollution. Vanderbilt Univ., Tennessee, EE.UU.

Humason, G.L. 1979. Animal Tissue Techniques. Freeman, San Francisco, California, EE.UU.

Johnson, R.A. \& D.W. Wichern. 1982. Applied multivariate statistical analysis. Prantice-Hall, Nueva Jersey, EE.UU.

Kinne, O. 1963. The effects of temperature and salinity on marine and brackish water animals. Oceanogr. Mar. Biol. 1: 301-340.

Kinne, O. 1964. The effects of temperature and salinity on marine and brackish water animals. Oceanogr. Mar. Biol. 2: 281-339.

Le Pennec, P., G. Benninger, G. Dorange \& P.M. Yves. 1991. Trophic sources and pathways to the developing gametes of Pecten maximus (Bivalvia:Pectinidae). J. Mar. Biol. 451-463.

Leyva-Valencia, L., A.N. Maeda-Martinez, M.T. Sicard, I.
Roldan, \& M. Robles-Mungaray. 2001. Halotolerance, upper thermotolerance, and optimum temperature for growth of the penshell Atrina maura (Sowerby, 1835) (Bivalvia: Pinnidae). J. Shellfish Res. 20: 49-54.

Loosanoff, V.L. \& H.C. Davis. 1952. Temperature requirements for maturation of gonads of northern oysters. Biol. Bull. (Woods Hole) 98: 60-65.

Loosanoff, V.L. \& H.C. Davis. 1963. Rearing of bivalve mollusk. p. 2-136. In F.S. Rusell (ed.) Advance in Marine Biology. I. Academic. Nueva York, Nueva York, EE.UU.

Lora-Vilchis, Ma. C., Ma. C. Rodríguez-Jaramillo \& A.N. Maeda-Martínez. 1997. Spawning process in the catarina scallop Argopecten ventricosus (Sowerby, 1842): Histological description. Eleventh international pectinid worshop. La Paz, Baja California Sur, México.

Mackie, G.L. 1984. Bivalves, p. 351-418. In Mackie G.L. (ed.). The Mollusca, reproduction. Academic, Nueva York, Nueva York, EE.UU.

Marshall, N. 1960. Studies on the Niantic River, Connecticut, with special reference to the Bay scallop Aequipecten irradians. Limnol. Oceanogr. 5: 85-105.

McWhinne, M.A. 1967. The heat responses of invertebrates. In A.H. Rose (ed.) Thermobiology. Academic. Nueva York, Nueva York, EE.UU.

Noguera de Gómez, O.\& S. Gómez-Aguirre. 1972. Memoria IV Congreso Nacional de Oceanografía. Ciclo sexual de Pinna rugosa Sowerby 1835 (Lamellibranchia: Pinnidae) en la Paz, Baja California Sur, México.

Orton, J.H. 1920. Sea temperatura, breeding and distribution of marine animals. J. Mar. Biol. Ass. U. K. 12: 339-366.

Prado, A.D. 1998. Ciclo Reproductivo de tres poblaciones de Chione californiensis Broderip, 1835 (Bivalvia: Veneridae) B.C.S., México. Tesis de Maestría, CICIMAR, La Paz, Baja California Sur, México

Rodríguez-Jaramillo, C., A.N. Maeda-Martínez, M.E. Valdez, T. Reynoso-Granados, P. Monsalvo-Spencer, D. Prado-Ancona, F. Cardoza-Velasco, M. RoblesMungaray \& M. T. Sicard. 2001. The effect of temperature on the reproductive maturity of the penshell Atrina maura (Sowerby, 1835) (Bivalvia: Pinnidae). J. Shellfish Res. 20: 39-47.

Santiago, C.J. 1981. Estudio gonadal de Pinna rugosa 
en la Bahía de la Paz, B.C.S. Tesis de Licenciatura, Universidad Autónoma de Baja California Sur, Baja California Sur, México.

Sastry, A.N. 1963. Reproduction in the Bay Scallop, Aequipecten irradians Lamarck. Influence of temperature on maturation and spawning. Biol. Bull. (Woods Hole) Mass. 25: 146-153.

Sastry, A.N. 1968. Relationship among food, temperature and gonad development of the bay scallop Aequipecten irradians Lamarck. Phys Zool. 41: 44-53.

Sastry, A.N. 1970. Reproductive physiological variations in latitudinally separated populations of the Bay Scallop Aequipecten irradians Lamarck. Biol. Bull. (Woods Hole) Mass. 138: 56-65.

Sastry, A.N. 1979. Pelecypoda (excluding Ostreidae), p. 113-292. In A.C. Giese \& J. S. Pearse (eds.). Reproduction of marine invertebrates. Academic, Nueva York, Nueva York, EE.UU.

Serrano-Guzmán, S.J. \& M. Robles-Mungaray. 1996. Informe Técnico 004-CREMES, 230196/1994. Cultivo de larvas y producción de larva fijadora de Atrina maura (Sowerby, 1835) del Golfo de California, México, en el Centro Reproductor de Especies Marinas del Estado de Sonora (C.R.E.M.E.S), Bahía de Kino, Sonora, México.

Serrano-Guzmán, S.J. 1997. Influencia de la temperatura en el crecimiento de juveniles de moluscos bivalvos nativos del Pacífico Mexicano. III Congreso Latinoamericano de Malacología (III CLAMA) y VI Reunión Nacional de Malacología y Conquiliología (VI RENAMAC). Ensenada, Baja California, México.

Soria, P.G. 1989. Aspectos poblacionales y datos preliminares para la evaluación del Callo de Hacha Atrina maura (Sowerby, 1835) en la desembocadura del río Balsas, Lázaro Cárdenas, Michoacán, México. Tesis de Licenciatura, Universidad Michoacana de San Nicolás de Hidalgo, Michoacán, México.

Vernber, F.J. 1962. Comparative physiology: latitudinal effects on physiological properties of animals. Ann. Rev. Physiol. 25: 517-546.

Walne, P.R. 1970. The seasonal variation of meat and glycogen content of seven populations of oyster Ostrea edulis L. and a review of the literature. Fish Invest. Ser. II. Mar. Fish. Great Britain. Minist. Agric. Fish. Food. 14. 43 p.

Yongqiang F. \& Xiang Q. 1988. Studies on ultraestructure of oocyte in process of maturing in pen shell Atrina pectinata. Acta Oceanologia Haiyang-Xuebao 7: $459-472$. 